\title{
Use of the Spine AdVerse Events Severity (SAVES) System to Categorize and Report Adverse Events in Spine Surgery.
}

\author{
Wagner $\mathrm{PJ}^{1}$, Ailon $\mathrm{T}^{2}$, Zhou $\mathrm{H}^{1}$, Pascal $\mathrm{S}^{1}$, Harrigan $\mathrm{MB}^{1}$, Stauff $\mathrm{M}^{1}$, Lapinsky $\mathrm{A}^{1}$, Connolly $\mathrm{PJ}^{1}$, \\ DiPaola $\mathrm{CP}^{1}$ \\ ${ }^{1}$ UMass Memorial Healthcare, University of Massachussetts Medical School, Worcester MA \\ ${ }^{2}$ Harvard School of Public Health, Boston MA \\ Contact information: Peter Wagner, peter.john.wagner@gmail.com, 631-848-8789
}

\section{Introduction}

Analysis of adverse events (AEs) in spine surgery has historically been retrospective, utilizing hospital administrative data. Our objective was to determine the incidence, severity and effect on hospital length of stay (LOS) for AEs in spine surgery using the Spine AdVerse Events Severity (SAVES V2) system.

\section{Methods}

AEs for all surgical spine patients at our institution were prospectively collected for 18 months and correlated with retrospective data from operative reports and H\&Ps. Statistical analyses compared patient demographics, diagnoses, and surgical characteristics to hospital length of stay and likelihood of adverse events.

\section{Results}

This system captured 75\% (765/977) of surgical cases for all indications over the study period. $73 \%(541 / 743)$ of patients experienced at least one AE, with an average of $1.2 \mathrm{AEs}$ per patient (range $0-5)$. The most common AEs were pain control (31\%), urinary retention (9.7\%), wound infection (6.3\%), and incidental durotomy (5.8\%). For patients experiencing at least one AE, $30 \%$ had no effect on LOS, $48 \%$ increased LOS by $1-2$ days, $15 \%$ increased LOS by $3-7$ days, and $7 \%$ had prolonged LOS greater than 8 days. Our system captured $25.4 \%$ more adverse events (60.0\% vs. $34.6 \%)$ than hospital administrative data. Univariate analysis revealed patient age, emergent surgery, diagnostic and surgical categories, and spine region to be predictors of both AEs and LOS. Instrumentation was predictive of increased LOS but not AEs. The type of AE was strongly associated with LOS. Multivariable analysis of AE likelihood demonstrated emergent surgery to be the strongest independent predictor with an adjusted odds ratio of 8.5 versus elective surgery.

\section{Discussion}

Spine surgery is associated with a high incidence of adverse events, which often prolong hospital length of stay. Better characterization of adverse events and their predictors could lead to improved management strategies that reduce patient morbidity and mortality. 